\title{
School and teacher factors associated with frequency of ICT use by mathematics teachers: Country comparisons
}

\author{
Willem J. Pelgrum • Joke Voogt
}

Published online: 23 June 2009

C The Author(s) 2009. This article is published with open access at Springerlink.com

\begin{abstract}
This paper explores school and teacher factors that are associated with the frequency of use of ICT by mathematics teachers. The SITES 2006 data base was used to compare countries with a relative high percentage of frequently ICT-using mathematics teachers (HIMA countries) with countries with a relative low percentage of frequently ICT-using mathematics teachers (LOMA countries). Meaningful effect sizes $(\leq-0.5$ or $\geq 0.5)$ for $29 \%$ of the items in the SITES 2006 database were found. The findings suggest that mathematics teachers in HIMA countries, more than in LOMA countries apply a learner-centered approach in their educational practice and have a focus on life long learning competencies. In addition, compared to LOMA countries, school leaders in HIMA countries, are active in stimulating the use of ICT and encouraging teachers to apply new ways of teaching and learning. The school level items of the SITES 2006 database with meaningful effect size were factor-analyzed resulting in five factors: 1 . active communication, 2. school leadership development, 3. assessment orientation, 4. ICT use by school leaders and 5 . Bottom-up change orientation. Country profile scores for HIMA and LOMA countries and for culturally comparable countries were generated to illustrate how these country profiles can be used to inform ICT policy.
\end{abstract}

Keywords Secondary analysis $\cdot$ Educational policies $\cdot$ ICT use $\cdot$ Mathematics · Implementation of ICT

\section{Introduction}

Since the mid 1980s Information and Communication technology (ICT) has been introduced in many education systems throughout the world. An observation from earlier international assessments of IEA (e.g. Pelgrum and Anderson 1999; OECD

\footnotetext{
W. J. Pelgrum $\cdot$ J. Voogt $(\bowtie)$

University of Twente, Faculty of Behavioural Sciences, P.O. Box 217, 7500 AE Enschede, The Netherlands

e-mail: j.m.voogt@utwente.nl
} 
2006) as well as many national assessments (e.g. Becker et al. 1999; Kennisnet 2008) was that the infusion of ICT in the daily learning practices of students was progressing very slowly and sometimes even stagnating. For policy makers, who have invested huge budgets in making ICT available for schools, major questions are why the progress is so slow and which barriers have to be removed in order to stimulate teachers to use new technologies in their educational practices. In this contribution we approach this question from theories about the implementation of educational change. Fullan (2007) in his most recent review of research on the implementation of educational change distinguishes three interactive factors affecting the implementation of educational change: 1 . characteristics of the change itself; 2. local characteristics (the role of the principal and the teacher in particular) and 3. external factors, referring to the role of governments. Ten Brummelhuis and Kuiper (2008) present a conceptual framework for understanding factors affecting the implementation of ICT. Their factors are more or less similar to the factors distinguished by Fullan. Key in their conceptual framework is the interaction between learner, teacher, content and (ICT) infrastructure and the effect of this interaction on the learning process. This interaction is affected by the school environment (the school leadership in particular) and the local environment, referring to the impact of local, regional and national policy.

The implementation of ICT can be seen as a complex innovation, mainly because the use of ICT is not only the introduction of a new tool in education. The integration of ICT in educational practice has the potential to facilitate new pedagogical approaches, learner-centered educational practices in particular (e.g. Dede 2000; AACTE 2008) and different learning outcomes, such as lifelong learning competencies. Life long learning competencies are considered important learning outcomes in the 21 st century (e.g. Anderson 2008). According to the European Commission, for instance, life long learning competencies include digital literacy (such as (internet) communication skills and information handling skills) and higher order skills such as teamwork, problem solving and project management (European Commission 2002). For ICT integration to be successful coherent efforts from school management and teachers are assumed. Newmann, King and Youngs (2000) found that coherence of innovations, technical resources and principal leadership need to be in place for schools to have the capacity to cope with educational change. According to Leithwood, Louis, Anderson and Walhlstrom (2004) successful principal leaders are engaged in setting (ambitious) goals and clear directions (vision building), are encouraging and supporting teachers to develop themselves, provide the necessary technical resources and build collaborative cultures. The latter also implies that school leaders actively communicate with about their plans teachers and parents. According to Dexter (2008) ICT leadership can be successful when school management sets clear learning goals that can be accomplished with the help of technology, put in place an ICT support system and create a learning environment in the school to develop teachers' technology skills and ICT-supported pedagogical skills. In addition, the school organization needs to be structured in such a way that teachers are supported in the pedagogical use of technology in their teaching practice. McLaughlin and Talbert (2001) also emphasize the need for teachers' participation in professional development activities as an integrated part of a collaborative culture in schools and supported by the principal as 
a condition for successful implementation of an innovation. Geijsel, Sleegers, Van den Berg and Kelchtermans (2001) found that teachers' participation in professional development activities contributed to teachers' implementation of the innovation in their classroom practice. Coherent efforts from school management and teachers to implement ICT in education need to be supported by national governments. Fullan (2007) mentions three important roles of national governments in large scale innovations: accountability, incentives and capacity building. According to Fullan the latter, capacity building, is often neglected in large scale innovations. Capacity building with a focus on results is needed to foster implementation of change, and this can be realized when bottom-up and top-down implementation strategies are both in place.

The conceptual framework that has been developed for SITES 2006 (see this issue's editorial introduction) has been based on theories of educational change as outlined above. The framework is based on the notion that teaching/learning practices as well as student outcomes may change as a result of the implementation of ICT in schools. In this paper we explore to what extent characteristics of the change, a learnercentered pedagogical approach and a focus on life long learning competencies in particular, as well as teacher and school factors are associated with frequent and nonfrequent use of ICT by mathematics teachers using the SITES 2006 database. The SITES 2006 database does not allow for an in-depth exploration of national policy data related to (non-)frequent use of ICT by mathematics teachers. For this reason national ICT policies are not part of the analysis carried out in this paper.

To investigate factors affecting the implementation of ICT, constructs were developed for the school and teacher level, as well as constructs characterizing the change itself:

- School factors: school leadership activities such as encouragement by school leaders of particular pedagogical practices, technical and pedagogical support, available ICT infrastructure, staff development requirements.

- Teacher factors: Technical/pedagogical competences; vision, availability and participation in professional development practices; perceived obstacles; presence of community of practice.

- Characteristics of the change factors: Curriculum goal orientation; teacher practice orientation; student practice orientation; assessment practice, learning resources; organization of teaching and learning; perceived impact of ICT.

These constructs were operationalized in indicators that were measured via questionnaires for school authorities (school leaders and technology coordinators) and teachers. The questionnaires together contained 550 items.

\section{Framing the research question}

Voogt (2008) in her analysis of a part of the SITES 2006 data showed that large differences existed between education systems ${ }^{1}$ in the extent to which mathematics

\footnotetext{
${ }^{1}$ Although in the SITES 2006 final report consistently the term 'education system' is used (because some countries participated that had more than one education system, e.g. Canada-Ontario and Canada-Alberta), for the sake of convenience we will use in this paper the term 'country'.
} 
and science teachers used ICT frequently (defined as 'at least once a week') for teaching/learning purposes. The focus of this paper is on mathematics teachers. Figure 1 provides an overview of the percentage of mathematics teachers that reported to frequently use ICT in their teaching practice.

In some education systems only a low proportion of the math teachers used ICT frequently: Chinese Taipei-7\%, Japan-3\%, Slovenia-7\%. This group of education systems will further on be called LOMA, while in other education systems this was much higher (HIMA): Chile-37\%, Canada-Ontario-43\%, Canada-Alberta-30\%. With regard to the national context indicators (such as ICT specific requirements for teacher certification, number of new pedagogies using ICT) that were presented by Anderson and Plomp (2008), no systematic differences were found between the HIMA and LOMA countries.

The research question that this paper addresses is: 'How do HIMA countries differ from LOMA countries with respect to characteristics of the change (pedagogical approach and life long learning competencies) as well as teacher and school factors affecting the implementation of ICT?'. This question is relevant for policy makers and scholars, because it gives more insight in potential causes of stagnating integration of ICT in existing school subjects, such as mathematics. In the remainder of this paper, first the methodology for addressing this research question will be

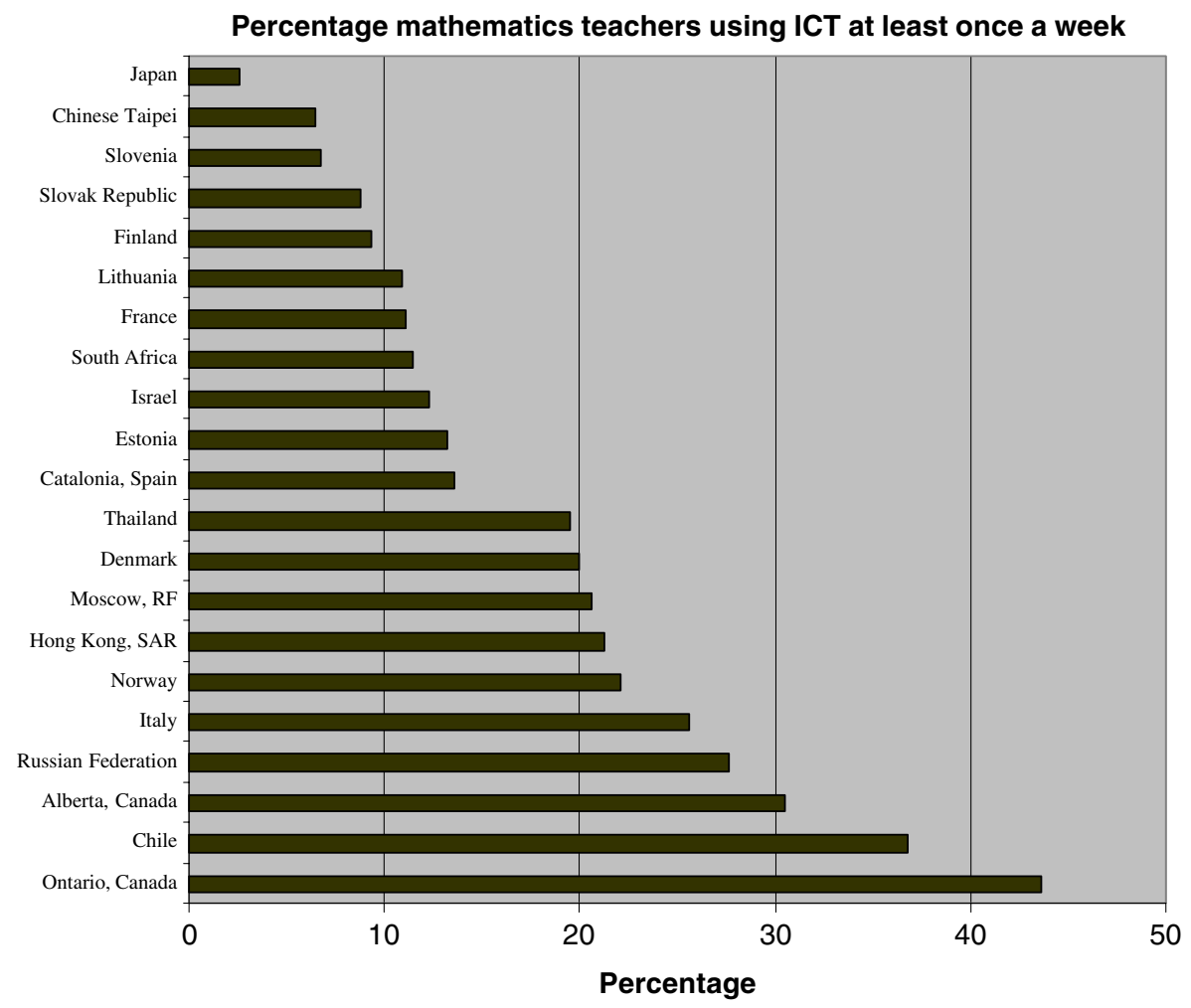

Fig. 1 Percentage of frequent ICT-using mathematics teachers per country. Note. Each bar represents the effect size for one item 
explained. This is followed by a description and discussion of the findings. The final section concerns conclusions and reflections about implications of the findings.

\section{Methodology and data sources}

The data sources that have been used for the comparison of HIMA and LOMA countries were the principal, technical coordinator and teacher survey data of SITES 2006 which are available through the web site of the IEA Data Processing and Research Centre (www.sites2006.net). While the purpose of this study was an open, explorative approach minimal restrictions have been put on measurement characteristics of the data and definitions of constructs. This implies that we did not want to be constrained by theoretical concepts and derived composite variables that were used in the SITES 2006 report (Law et al. 2008), but that we used a singleton approach by focusing on questionnaire items rather than composites. A main reason for using this singleton approach is that composite variables sometimes hide interesting phenomena that can be observed when examining the items underlying the composite.

The following example may help to illustrate this point. Based on the principal questionnaire, Pelgrum (2008) used a composite variable called 'presence of emerging pedagogy', consisting of a score based on six questionnaire items, reflecting independent and autonomous learning by students. It was observed that interesting differences existed when comparing the mean score per country between 1998 (SITES Module 1) and 2006, for instance in some countries the score declined substantially (e.g. Denmark and Norway), while in other countries substantial increases were observed (e.g. Hong Kong and Lithuania). However, when the comparison was done for each of the underlying items separately, it appeared that quite different patterns showed up, e.g. whereas the composite score for Denmark decreased a substantial increase showed up in one of the underlying items ('students learn to search for, process and present information').

For the analysis of differences between the two groups of countries in principle all questionnaire items from SITES 2006 (principal questionnaire, technical questionnaire and teacher questionnaire) have been used. A number of SITES 2006 variables have not been included, because they were considered not to be relevant for the purpose of this article, viz. to acquire more insight in potential causes of stagnating integration of ICT (e.g. the total number of students in the school,). The set that is used for the analysis covers nearly $90 \%$ of all SITES 2006 variables. A number of items had to be recoded, before they could be used for our analysis. For instance:

- A question in the technical questionnaire about the availability of technology applications, for which the answer options were: 'available', 'needed but not available', 'not needed and not available'. As there are two aspects covered by the answer codes (availability and need), the codes for these answer options were recoded in order to reflect a distinction between available and not available.

- A question in the teacher questionnaire about participation in professional development activities, for which the answer options were: 'No, I don't wish to attend'; 'No, I would like to attend if available'; 'Yes, I have'. The answer codes 
were recoded in order to reflect a distinction between 'Attended' and 'Not attended'.

For interpreting the size of differences between the two groups of countries we will use statistics that is known as Cohen's effect size $d^{2}$ (Cohen 1992). Cohen provided the following tentative guidelines for the interpretation of effect sizes: small effect (absolute value $d \leq 0.2$ ), medium (absolute value $d$ about 0.5 ) and large (absolute value $d \geq 0.8$ ). This means that roughly one standard deviation difference (as compared to the average standard deviations in both groups) is considered to be a substantial difference. For the purpose of this article we have used effect sizes larger than 0.5 or lower than -0.5 for qualifying differences between the two groups as meaningful. This step in the analysis has resulted in a list of variables (at school- and teacher-level) on which the HIMA countries differ positively or negatively from the LOMA countries. To develop a deeper understanding of the initial findings at the school level the 53 meaningful items of the school level data (based on the principal and technical coordinator survey) were further analyzed using factor analysis to reduce the data set.

\section{Initial findings}

\subsection{Overall findings}

The overall effect sizes of the 550 questionnaire items from the principal, technology coordinator and teacher questionnaire is shown in Fig. 2.

Figure 2 shows that the effect sizes of a large number of items were meaningful according to the criterion that was mentioned in the previous section $(\leq-0.5$ or $\geq 0.5)$. This concerned 158 of the 550 items (29\%). For national policy-making in the LOMA countries this list may serve as a first basis for reflection on potential interventions aimed at increasing the use of ICT in mathematics teaching and/or learning.

Furthermore we can infer from Fig. 2 that:

- Positive effect sizes were outnumbering the negative ones (26\% versus $3 \%)$.

- 35 items showed large positive effect sizes $(\geq 0.8)$. Large negative effect sizes hardly occurred (only on two variables).

The following are a few examples of items for which relatively large effect sizes were observed:

- School leaders inform teachers about pedagogical changes taking place in the school $(d=0.9)$

- School leaders motivate teachers to critically assess their own educational practices $(\mathrm{d}=1.1)$

- Teachers use ICT for extended projects $(\mathrm{d}=1.1)$

- Teachers indicate that their priority in the next years is to involve students in extended collaborative projects $(\mathrm{d}=1.1)$

\footnotetext{
${ }^{2}$ Cohen's effect size: $\mathrm{d}=\left(\right.$ mean $\left._{1}-\mathrm{mean} 2\right) /\left(\sqrt{ }\left(\mathrm{SD}_{1}{ }^{2}+\mathrm{SD}_{2}{ }^{2}\right) / 2\right)$
} 
Fig. 2 Comparison between HIMA and LOMA countries for 550 questionnaire items

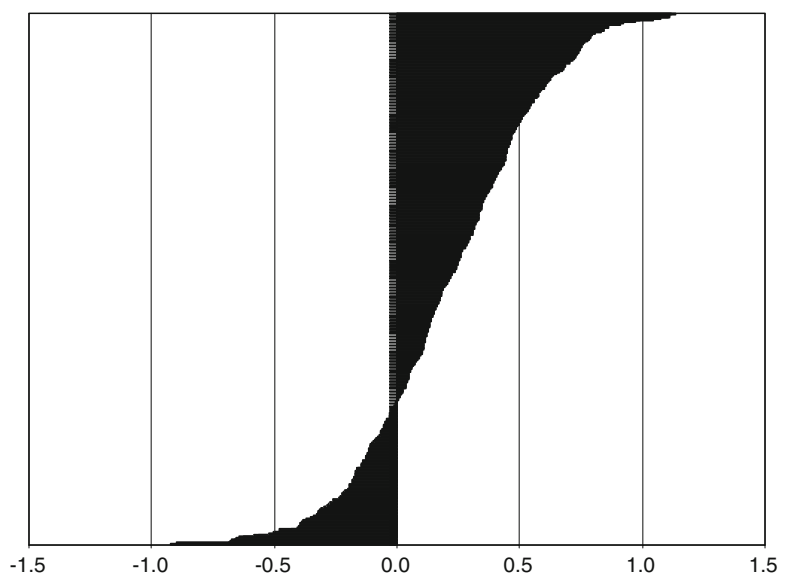

- Teachers use ICT during a limited period of time $(\mathrm{d}=-0.9)$

- School principals encourage teachers to collaborate with teachers from other countries $(\mathrm{d}=-0.9)$

A more extensive description of all observed differences is presented in the following section. First a description will be given of how HIMA and LOMA countries differed in terms of school level conditions (as reported by principals and technology coordinators). This is followed by a summary of differences that were observed at the teacher level.

\subsection{School level}

From the 284 school level items that were included in the analysis 53 showed meaningful effect sizes. With regard to the characteristics of the change, the pedagogical approach and the focus lifelong learning competencies, school principals in HIMA countries reported more-as compared with principals in LOMA counties - that in their schools students were:

- developing abilities to undertake independent learning;

- learning to search for, process and present information;

- learning and working during lessons at their own pace.

With regard to the organization of learning, school leaders in HIMA countries reported more often that students work in different groups according to the projects that they are engaged in or the subjects they are taking, but they also indicated that changes to the usual time schedule occurred if students needed time to complete their projects. On the contrary, school leaders in LOMA countries indicated more often that students were following their lessons according to a fixed schedule.

Consistent with the group categorization for the selection of countries that were involved in the effect size calculation, is the observation that technology coordinators in HIMA countries indicated more often that their school had integrated ICT in most teaching and learning processes. They indicated that in particular ICT was used for the learning of mother tongue, mathematics and social 
sciences. On the contrary in the LOMA countries ICT seems to be more frequently used by students in a separate ICT-subject. Also computers are, according to technology coordinators in HIMA countries, more often available in most classrooms.

In addition the results of the exploration suggest that school leaders in the HIMA countries, more than their colleagues in the LOMA countries tend to set directions for using ICT. Teachers were encouraged to cover (in general) the prescribed curriculum content, to assign production projects to students and to prioritize resource allocations to improve students' ICT skills. They also encouraged teachers to use particular forms of assessment, such as: written tests/examinations, written tasks/exercises and oral and/or written group presentations. School principals in HIMA countries were also more inclined to actively monitor and evaluate the implementation of pedagogical changes.

School leaders in HIMA countries were much more active in undertaking communicative activities in their school. Items with effect sizes larger than 0.8 were:

- informing teachers about pedagogical changes taking place in the school;

- informing teachers about educational developments outside the school;

- motivating teachers to critically assess their own educational practices;

- discussing with parents and students the pedagogical changes taking place in the school.

In HIMA countries school leaders reported more often that they used ICT for communication with teachers and parents, and also for budgeting, monitoring and controlling expenses and/or time tabling.

School principals in HIMA countries also assigned higher priorities to acquire, as school leaders, competencies with regard to:

- explaining teachers the relevance of encouraging students to be responsible for their own learning and outcomes;

- identifying best practices existing outside the school regarding the integration of ICT in learning;

- promoting collaboration between teachers of different subjects;

- managing the adoption of ICT-supported methods for assessing students progress;

- promoting the integration of ICT in the teaching and learning of subjects.

HIMA and LOMA countries hardly differed in terms of obstacles that were perceived as hindering the schools' capacity to realize its pedagogical goals, except that in HIMA countries it has been more often reported that there were insufficient numbers of computers connected to the Internet.

Finally, with regard to background characteristics of schools it appeared that absenteeism of students in HIMA countries was slightly higher than in LOMA countries.

Whereas above mainly a description was given of the items on which the HIMA countries scored higher (with here and there some mentioning of the LOMA countries), it further appeared that the LOMA countries scored higher $(\mathrm{d} \leq-0.5)$ on, for example, the following items:

- providing teachers with laptop computers and/or other mobile devices;

- encouraging teachers to involving students in self-accessed courses and/or learning activities; 
- encouraging teachers to collaborate with teachers from other countries;

- availability of (non-ICT) equipment and hands-on materials;

- maintenance of hardware by external companies hired by the school;

- students spending their time in school following lessons according to a fixed schedule;

- students are more often learning about ICT in a separate subject;

- computers available in some classrooms;

- teachers acquired knowledge and skills in using ICT via reading professional journals and/or similar publications;

- teachers, parents and/or students initiated changes that were considered highly satisfying by school leaders.

These higher scores cannot easily be interpreted in terms of factors affecting the implementation of ICT as outlined above, except for the last two items. Teacher professional development in LOMA countries seems to be an individual endeavor and not a collaborative effort, which does comply with recent findings regarding the importance of in-school collaborative professional development to foster the implementation of educational change (McLaughlin and Talbert 2001). The last item suggest that school principals in LOMA countries favor a bottom-up approach to educational change, while recent literature on successful change strategies promote an combination of bottom-up and top-down approaches (Fullan 2007).

\subsection{Teacher level}

With regard to the items at the teacher level, it appeared that $40 \%$ of the 266 items had meaningful effect sizes. This number is too large for a detailed description as was given above for the school level items. Therefore, we will provide a more global summary. For the interested reader Table 1 in Annex 1 is included, showing the items (with variable names used in the SITES 2006 data base, see www.sites2006. net) that had absolute effect sizes $>0.5$. Because the HOMA and LIMA countries were selected based on the frequency of ICT use by mathematics teachers the HIMA and LOMA groups are not independent with regard to ICT variables. For this reason the description of the results on the teacher level data will focus only on the non-ICT variables.

What appears from an inspection of the non-ICT items is that-related to the characteristics of the change (a learner-centered educational approach as well as a focus of life long learning competencies) — in HIMA countries, for instance:

- teachers assigned more importance to activities promoting life long learning competencies;

- students were more engaged in determining their own content goals, explaining and discussing own ideas with teachers and peers, and self and/or peer evaluation;

- there is more flexibility in terms of the locations where students work.

Also, consistent with what school leaders in HIMA countries indicated, more mathematics teachers in HIMA countries, compared to mathematics teachers from LOMA countries, reported that the vision of the school staff was to constantly 
motivate teachers to critically assess their own educational practices and to think about the vision and strategies of the school. Mathematics teachers also felt that they could influence innovation plans in the school and that they were able to implement innovations in their classroom according to their own insights and judgments. These results suggest that in HIMA countries more than in LOMA countries teachers experience a collaborative school culture.

With regard to background variables, in HIMA counties the number of mathematics lessons per week was higher than in LOMA countries and the absenteeism of students was higher. The latter was also reported by school leaders in HIMA countries.

\section{Country profiles}

The next step of the analysis was based on the principal and technical coordinator data. For this analysis we used those school level items (based on the principal and technical coordinator questionnaires) that showed meaningful effects sizes. As described above, it appeared that 53 items showed absolute effect sizes that were larger than 0.5 . Hence we explored whether this set could be reduced through factor analysis in a meaningful way. To find robust factors we included all SITES 2006 countries in the factors analysis. It appeared that a set of five interpretable factors could be identified. These factors (and a short description of the underlying items) were labeled as follows:

1. Active communication within school, consisting of items, such as: informing teachers and parents about pedagogical changes, discussing with teachers and students about teaching and learning.

2. Priorities for school leadership development, consisting of items indicating priorities for the school leadership to acquire skills in change management.

3. Assessment orientation, that is school leaders encourage teachers to use different types of assessment for monitoring student progress.

4. ICT use by school leaders, for communication and administrative activities.

5. Bottom up change-orientation, implying that satisfying changes in the school had been initiated by teachers and/or students.

These factors are well interpretable and can be related to the implementation of educational change as a characteristic of school leaders. Active communication is an essential activity in developing a collaborative culture (factor 1). Factor 2 and 4 can be conceived as a necessary condition for realizing, respectively facilitating leadership goals. Factor 5 seems an indication of an approach that discourages the implementation of educational innovations, as research shows that a combination of bottom-up and top-down is preferable (Fullan 2007). Factor 3 is more difficult to interpret as factor affecting educational change. Although the literature is quite clear on the need for school principals to set clear learning outcomes; the literature does not tell much about the need for variation in forms of assessment for assessing student learning outcomes. However it is obvious that ICT offers possibilities for more variation in forms of assessment. 
The factor scores for each factor were calculated using the regression method. In this way standardized scores were acquired with a mean of zero and a standard deviation of 1 . The minimum and maximum scores for each of the factors varied between -2.8 and 3.6 for factor $1,-4.3$ and 2.6 for factor $2,-4.7$ and 2.2 for factor $3,-2.6$ and 2.7 for factor 4 and -2.0 and 2.4 for factor 5 .

In order to illustrate how these factor scores might be used for policy reflections a number of comparisons between arbitrary chosen pairs of HIMA and LOMA were conducted, resulting in country profiles. In addition, two comparisons will be shown for countries that are culturally comparable and hence are expected to result in comparable profiles. Below we will describe the outcomes of these profile analyses and their potential implications for policy-making.

Figure 3 contains the profile of a HIMA country (in this case Chile with $37 \%$ mathematics teachers using ICT frequently) and a LOMA country (Chinese Taipei, $7 \%$ frequent use). From Fig. 3 one may infer that in Chinese Taipei, in comparison with Chile, school leaders are less active in communicating about pedagogical changes (factor 1) and also have lower priorities for developing their change management competencies (factor 2). On the other hand it seems that in Chinese Taipeh the initiative for realizing changes is much more left to teachers and/or students (factor 5). This could mean that policies for stimulating a more active school leadership in initiating ICT-related changes might be worthwhile to consider in Chinese Taipei. It is not known whether these different profiles result from differences in national strategies for integrating ICT in teaching and learning. Nevertheless, it seems warranted to advise policy-makers in Chinese Taipei to reflect further on these strategies and to determine whether something can be learned from the strategies applied in Chile.

Another illustration of a profile comparison of a HIMA (Canada-Ontario with $30 \%$ frequent use) and a LOMA country (Japan with 3\% of frequent use) is shown in Fig. 4.

The profiles in Fig. 4 are quite different from the ones in Fig. 3. Canada-Ontario stands out in the use of ICT by school leaders (factor 4). In terms of active communication of school leaders about pedagogical changes (factor 1) and in the

Fig. 3 Scores on five factors for Chile (HIMA) and Chinese Taipeh (LOMA). Note: Factor 1: Active communication, Factor 2: Priorities for school leadership development, Factor 3: Assessment orientation, Factor 4: ICT use of school leader, Factor 5: Bottom-up change orientation

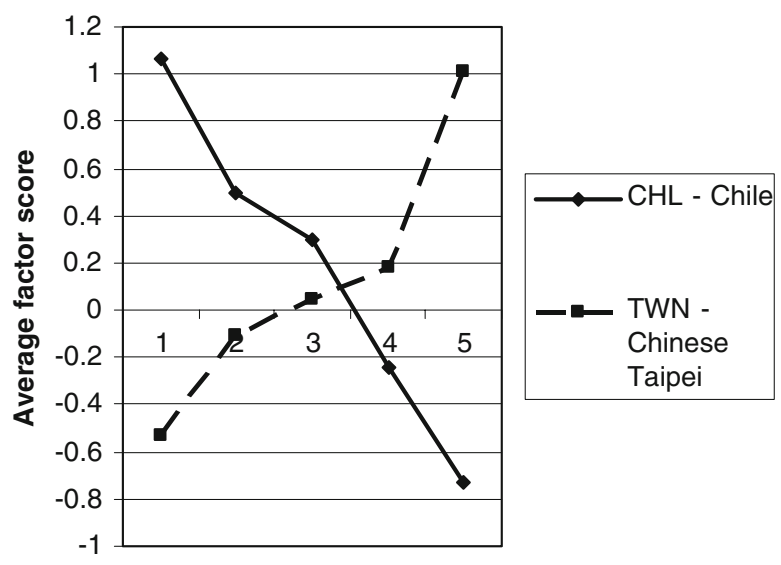


Fig. 4 Scores on five factors for Canada-Ontario (HIMA) and Japan (LOMA). Note: Factor 1: Active communication, Factor 2: Priorities for school leadership development, Factor 3: Assessment orientation, Factor 4: ICT use of school leader, Factor 5: Bottom-up change orientation

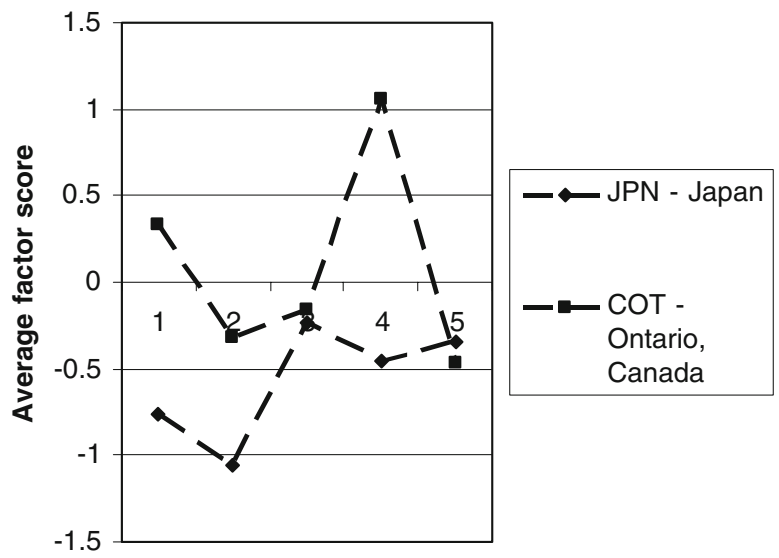

need for school leadership development (as perceived by school leaders themselves) (factor 2) Canada scores substantially higher than Japan.

Important for policy-makers, when using this kind of profile analysis is to first determine which countries are relevant to compare with. Arguments like economical competitiveness might for instance play a role in such deliberations. Next, the analysis might be used for generating ideas on potential levers for change.

Whereas the profiles in the comparison above are quite different, it is interesting to explore whether more similarity appears, if the comparison is done for countries that are more or less culturally comparable. Such a comparison also contributes to the validation of the findings. For this comparison two systems from the same country (in this case the Russian Federation and Moscow, for which the data sets were independent, Fig. 5) are compared as well as two different countries from the same region (Denmark and Norway, Fig. 6).

In Fig. 5 it is clear that the profiles of Moscow and the total Russian Federation are almost identical. These are characterized by positive scores on active communication, (factor 1), school leadership development needs (factor 2) and

Fig. 5 Scores on five factors for the Russian Federation and Moscow. Note: Factor 1: Active communication, Factor 2: Priorities for school leadership development, Factor 3: Assessment orientation, Factor 4: ICT use of school leader, Factor 5: Bottomup change orientation

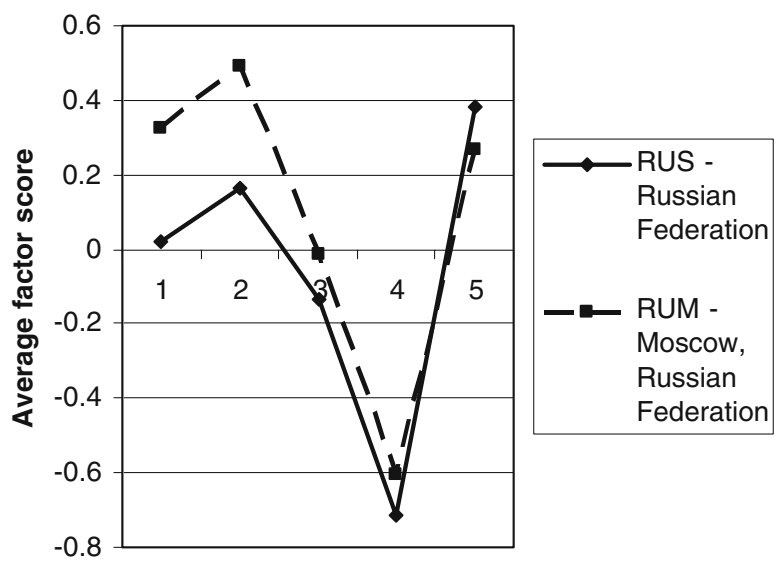


Fig. 6 Scores on five factors for the Denmark and Norway. Note: Factor 1: Active communication, Factor 2: Priorities for school leadership development, Factor 3: Assessment orientation, Factor 4: ICT use of school leader, Factor 5: Bottom-up change orientation

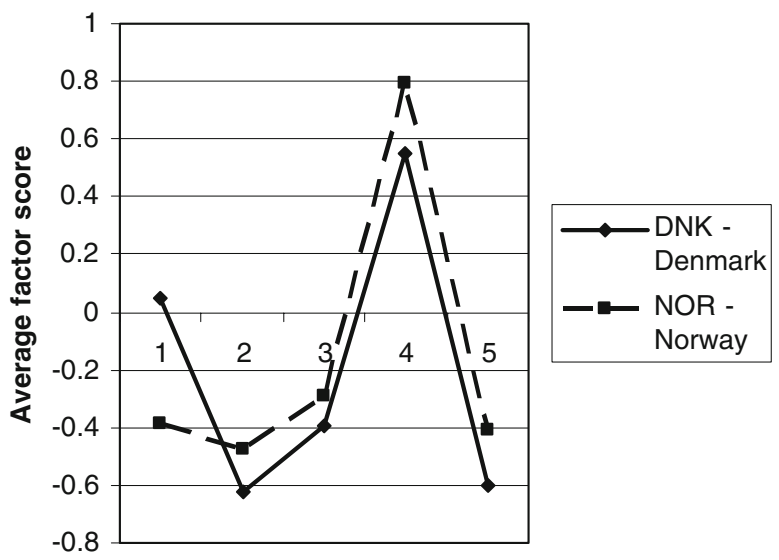

bottom-up orientation factor 5), no clear emphasis on forms of assessment (factor 3), and very low use of ICT by school leaders (factor 4). When comparing two other systems from the same country (Canada-Ontario and Canada-Alberta) it was also observed that the profiles were nearly identical.

When comparing two different countries from the same region (Denmark and Norway) we can also observe (Fig. 6) nearly identical profiles. It seems that in these two countries many school leaders use ICT themselves (factor 4), but the sores on the other factors are not very meaningful.

\section{Conclusions, reflections and implications}

The results of the analyses presented above show that the differences between HIMA and LOMA countries with regard to school and teacher factors are quite well interpretable. The findings suggest that mathematics teachers in HIMA countries, more than in LOMA countries, apply a learner-centered approach in their educational practice and have a focus on life long learning competencies. School leaders in HIMA countries do not seem to ignore the need for covering the content of the prescribed curriculum, but in addition they provide room for other goals and seem to encourage new pedagogical approaches to realize this. The results also indicate that in HIMA countries, more than in LOMA countries, school leaders are active in stimulating the use of ICT, encouraging teachers to apply new ways of teaching and learning. In addition more school leaders in HIMA countries, compared to LOMA countries, support teachers' development of ICT skills and ICT-supported pedagogical skills and create a collaborative school culture that supports the implementation of ICT. According to the mathematics teachers, school leaders in HIMA countries actively communicate their pedagogical vision with teachers and parents. The findings from the exploration we conducted are consistent with other studies on educational change (Dexter 2008; Fullan 2007; Leithwood et al. 2004; McLaughlin and Talbert 2001).

The work reported in this paper is quite exploratory. Further analyses are needed to determine which refinements are possible. Probably further fine-tuning is possible with 
regard to the cut-off criteria that were used for selecting items with meaningful effect sizes. We used as lower threshold an absolute effect size of 0.5 , but maybe the results would still be meaningful and interpretable when this threshold would be lowered.

The analysis in this paper was based on the frequency of ICT use by mathematics teachers. In addition to country comparisons as have been studied in this paper, we would also suggest to further investigate school and teacher factors affecting the implementation of ICT within a country. Whereas the analyses in this paper were based on mathematics teachers, future work will also focus on investigating on which school and teacher variables countries with frequent use of ICT in science lessons differ from countries with non frequent use of ICT in science education. Moreover, more work is needed with regard to national context variables. What needs to be sorted out is to what extent the HIMA and LOMA countries differ in terms of the characteristics - from the perspective of national factors affecting educational change - of specific national policies to integrate ICT in the teaching and learning of mathematics.

The comparisons of profiles between specific countries do not immediately lead to recipes for policy planning. The comparisons presented here rather could function as lever for raising awareness at the policy level and generating questions, such as: if we think that the integration of ICT in the teaching and learning of mathematics is important how can school leaders be activated to play a role in realizing such intentions? In discussing such questions, one may decide to look for (good) practices in other countries. For instance, the 'Enlaces Project' in Chile may be a good model that might be considered by policy makers in other countries. Important factors in the success of the Enlaces Project are its focus on teachers and teaching (and not the technology), the use of an existing infrastructure, decentralized technical assistance and training and a robust system for monitoring progress (see, for instance learnlink.aed.org/Publications/ Sourcebook/chapter4/chile_casestudy.pdf). The analyses shown in this paper indicate that the SITES 2006 data base is useful as starting point for data-driven policy making with regard to the integration of ICT in education.

\section{Annex 1}

Table 1 Teacher items with absolute effect sizes $\geq .5$ (variable names as used in the SITES2006 data base: www.sites2006.net)

\begin{tabular}{llllllll}
\hline Var-name & Eff. Size & Var-name & Eff. Size & Var-name & Eff. Size & Var-name & Eff. Size \\
BTG04A1 & 0.5 & BTG14A1 & 0.9 & BTG17D1 & 0.5 & BTG22G1 & 1.1 \\
BTG06A1 & 0.9 & BTG14B1 & 0.6 & BTG17F1 & 0.6 & BTG22H1 & 0.8 \\
BTG07B1 & 0.5 & BTG14B2 & 0.7 & BTG17H1 & 0.6 & BTG22I1 & 0.7 \\
BTG07D1 & 0.7 & BTG14C1 & 0.6 & BTG18A1 & 0.7 & BTG22J1 & 0.8 \\
BTG07E1 & 0.8 & BTG14C2 & 0.6 & BTG19A1 & 0.5 & BTG22L1 & 0.5 \\
BTG07F1 & 0.8 & BTG14D1 & 0.8 & BTG19B1 & 0.5 & BTG25B1 & 0.7 \\
BTG08A1 & 0.7 & BTG14F1 & 0.6 & BTG19D1 & 0.6 & BTG25C1 & 0.6 \\
BTG08D1 & 0.8 & BTG14G1 & 0.5 & BTG19E1 & 0.6 & BTG26A1 & 0.7 \\
BTG08G1 & 0.8 & BTG14H1 & 0.8 & BTG19F1 & 0.5 & BTG26C1 & 0.5
\end{tabular}




\begin{tabular}{llllllll} 
BTG08H1 & 0.6 & BTG14I1 & 0.7 & BTG19G1 & 0.5 & BTG37A1 & -0.9 \\
BTG081 & 0.7 & BTG14L1 & 0.8 & BTG19H1 & 0.5 & BTG39E1 & 0.5 \\
BTG08L1 & 0.7 & BTG14L2 & 0.7 & BTG20A1 & 0.6 & BTG39K1 & 0.5 \\
BTG09A1 & 0.7 & BTG15B2 & 0.5 & BTG20C1 & 0.6 & BTG40N1 & -0.5 \\
BTG09A2 & 1.1 & BTG15C2 & 0.5 & BTG20G1 & 0.5 & BTG41J1 & 0.7 \\
BTG09B1 & 0.8 & BTG15D1 & 1.0 & BTG20H1 & 0.7 & & \\
BTG09B2 & 0.8 & BTG15D2 & 0.7 & BTG20J1 & 0.7 & & \\
BTG09C1 & 0.8 & BTG15E1 & 0.7 & BTG20L1 & 0.5 & & \\
BTG09C2 & 0.7 & BTG15E2 & 0.8 & BTG20M1 & 0.5 & & \\
BTG09D2 & 0.5 & BTG15F1 & 0.7 & BTG20N1 & 0.6 & & \\
BTG09E1 & 0.6 & BTG15H1 & 1.1 & BTG21I1 & 0.6 & & \\
BTG09E2 & 0.5 & BTG15H2 & 0.5 & BTG21J1 & 0.5 & & \\
BTG09F1 & 0.5 & BTG16A2 & 0.7 & BTG21L1 & 0.5 & & \\
BTG09H2 & 0.7 & BTG16B1 & 0.5 & BTG21M1 & 0.5 & & \\
BTG0912 & 0.5 & BTG16B2 & 0.8 & BTG21N1 & 0.5 & & \\
BTG09J2 & 0.6 & BTG16D2 & 0.7 & BTG21P1 & 0.9 & & \\
BTG09L1 & 0.8 & BTG16E1 & 0.6 & BTG22A1 & 0.7 & & \\
BTG09L2 & 0.9 & BTG16E2 & 0.5 & BTG22B1 & 0.6 & & \\
BTG09M1 & 0.9 & BTG16I1 & 0.5 & BTG22D1 & 1.0 & & \\
BTG09M2 & 0.6 & BTG17A1 & 1.0 & BTG22E1 & 0.7 & \\
BTG11A1 & 0.8 & BTG17B1 & 0.5 & BTG22F1 & 1.1 & \\
\hline
\end{tabular}

Open Access This article is distributed under the terms of the Creative Commons Attribution Noncommercial License which permits any noncommercial use, distribution, and reproduction in any medium, provided the original author(s) and source are credited.

\section{References}

AACTE Committee on Innovation and Technology. (2008). (Eds.). Handbook of technological pedagogical content knowledge (TPCK) for educators. New York/London: Routledge.

Anderson, R. (2008). Implications of the information and knowledge society for education. In J. Voogt \& G. Knezek (Eds.), International handbook of information technology in primary and secondary education (pp. 3-22). New York: Springer.

Anderson, R. E., \& Plomp, T. (2008). National contexts. In N. Law, W. J. Pelgrum \& T. Plomp (Eds.), Pedagogy and ICT use in schools around the world: Findings from the IEA SITES 2006 study (pp. 37-66). Dordrecht \& Hong Kong: Springer \& CERC.

Becker, H. J., Rawitz, J. L., \& Wong, Y. T. (1999). Teacher and teacher-directed student use of computers and software. Irvine, CA: University of California.

Cohen, J. (1992). A power primer. Psychological Bulletin, 112, 155-159. doi:10.1037/0033-2909.112.1.155.

Dede, C. (2000). Emerging influences of information technology on school curriculum. Journal of Curriculum Studies, 32(2), 281-303. doi:10.1080/002202700182763.

Dexter, S. (2008). Leadership for IT in schools. In J. Voogt \& G. Knezek (Eds.), International handbook of information technology in primary and secondary education (pp. 543-551). New York: Springer.

European Commission. (2002). eEurope 2005: An information society for all. Brussels, Belgium: European Commission.

Fullan, M. (2007). The new meaning of educational change (4th ed.). London: Falmer.

Geijsel, F., Sleegers, P., Van den Berg, R., \& Kelchtermans, G. (2001). Conditions fostering the implementation of large scale innovation programs in schools: Teachers' perspectives. Educational Administration Quarterly, 37, 130-166. doi:10.1177/00131610121969262. 
Kennisnet (2008). Four in balance monitor 2008. ICT at Dutch schools. Zoetermeer, NL: Kennisnet Foundation.

Law, N., Pelgrum, W.J., \& Plomp, T. (Eds.). (2008). Pedagogy and ICT use in schools around the world: Findings from the IEA SITES 2006 study. Dordrecht \& Hong Kong: Springer \& CERC.

Leithwood, K., Louis, K., Anderson, S., \& Walhlstrom, K. (2004). How leadership influences student learning. New York: Wallace Foundation.

McLaughlin, M., \& Talbert, J. (2001). Professional Communities and the work of high school teaching. Chicago: University of Chicago Press.

Newmann, F. M., King, M. B., \& Youngs, P. (2000). Professional development that addresses school capacity: lessons from urban elementary schools. American Journal of Education, 108(4), 259-299. doi:10.1086/444249.

Organisation for Economic Co-operation and Development. (2006). Are students ready for a technology rich world? What PISA studies tell us. Paris: OECD.

Pelgrum, W. J., \& Anderson, R. A. (eds). (1999). ICT and the merging paradigm for life-long learning: A worldwide assessment of infrastructure, goals and practices. Amsterdam: International Association for the Evaluation of Educational Achievement.

Pelgrum, W. (2008). School practices and conditions for pedagogy and ICT. In N. Law, W. J. Pelgrum \& T. Plomp (Eds.), Pedagogy and ICT use in schools around the world: Findings from the IEA SITES 2006 study (pp. 67-120). Dordrecht \& Hong Kong: Springer \& CERC.

Ten Brummelhuis, A., \& Kuiper, E. (2008). Driving forces for ICT in learning. In J. Voogt \& G. Knezek (Eds.), International handbook of information technology in primary and secondary education (pp. 97-111). New York: Springer.

Voogt, J. (2008). Satisfying pedagogical practices. In N. Law, W. J. Pelgrum \& T. Plomp (Eds.), Pedagogy and ICT use in schools around the world: Findings from the IEA SITES 2006 study (pp. 221-250). Dordrecht \& Hong Kong: Springer \& CERC. 